DESY 97-132

\title{
Quadratically optimized polynomials for fermion simulations
}

\author{
I. Montvay \\ Deutsches Elektronen-Synchrotron DESY, \\ Notkestr. 85, D-22603 Hamburg, Germany
}

\begin{abstract}
Quadratically optimized polynomials are described which are useful in multi-bosonic algorithms for Monte Carlo simulations of quantum field theories with fermions. Algorithms for the computation of the coefficients and roots of these polynomials are described and their implementation in the algebraic manipulation language Maple is discussed. Tests of the evaluation of polynomials on dynamical fermion configurations are performed. In a simple special case the obtained polynomial approximations are compared to Chebyshev polynomials.
\end{abstract}




\section{Introduction}

The numerical simulation of quantum field theories with fermions is an interesting and difficult computational problem. The basic difficulty is the necessary calculation of very large determinants, the determinants of fermion matrices, which can only be achieved by some stochastic procedure with the help of auxiliary bosonic "pseudofermion" fields (for general background see, for instance, [1]).

A promising new approach proposed by Lüscher [2] is based on polynomial approximations of some negative powers $x^{-\alpha}$ of the fermion matrix. In this case the number of auxiliary bosonic fields is equal to the order of the polynomial and the resulting multibosonic action can be treated by simple methods known from bosonic quantum field theories without fermions. The performance of such a multi-bosonic algorithm can be improved in a two-step polynomial approximation scheme [3], where the number of bosonic fields is equal to the order of a first, relatively low order, polynomial only realizing a modest approximation of $x^{-\alpha}$. The required high precision is achieved in a "noisy" correction step as proposed some time ago by Kennedy and Kuti [4]. In the correction step some high order polynomial approximations of $x^{-\alpha} / \bar{P}(x)$ are used, where $\bar{P}(x)$ is some polynomial. The necessary polynomials can be obtained by minimizing the integral of squared relative deviations in an interval containing the eigenvalue spectrum of the fermion matrix.

The computation of the quadratically optimized polynomial approximations of functions of general type $x^{-\alpha} / \bar{P}(x)$, which are required in the two-step multi-bosonic algorithms, has been briefly outlined in ref. [3]. It can be best done by using the arbitrarily high precision facilities of an algebraic manipulation program, as for instance Maple V. In the present paper the algorithms for the calculation of the coefficients and roots of these polynomials are described in more detail. The optimal ordering of the roots in applying the polynomials of the fermion matrix in a product form is also discussed. This is necessary in order to keep rounding errors tolerable even for 32 bit arithmetics.

These kinds of quadratically optimized polynomial approximations belong to the general class of "least-squares" or "Gaussian-" approximations [5]. The coefficients of the polynomials can be obtained, for instance, by expanding the function to be approximated in terms of suitably defined orthogonal polynomials. The expansion in orthogonal polynomials provides the possibility of a recursive evaluation without knowing the roots, which is advantageous from the point of view of rounding errors. In case if the roots of the approximating polynomials are also required, the calculation of the polynomial coefficients

is the smaller part of the calculation. The larger part is to determine the roots with sufficient precision and find their optimal ordering.

Another possibility, besides the quadratic optimization, is to consider "minimax" or "infinity-norm" approximation schemes, where the maximum deviation in the region of 
approximation is minimized [5]. For instance, in ref. [2] this has been used to approximate the function $x^{-1}$ by Chebyshev polynomials. In multi-bosonic algorithms, in general, the quadratic optimization seems more appealing, because it provides better approximations in the average. In addition, the advantage of the quadratic optimization is its great flexibility. For instance, besides the possibility to choose a wide class of functions, it also allows for arbitrary complex regions and general weight factors which also take into account the average eigenvalue distribution of the fermion matrix. Despite this generality, in all cases one can use essentially the same algorithm to determine the polynomials. Nevertheless, in the case of the function $x^{-1}$ the choice between minimax and least-squares approximation can only be decided by practical tests and the outcome may also depend on the preferences of a particular application. (The same applies to the generalization of the Chebyshev approximation to the more general case of $x^{-\alpha}$ which has been proposed recently by Bunk [6].)

The plan of this paper is as follows: In the next section first the definitions and some basic properties of the polynomials necessary in the first step of the two-step multi-bosonic algorithm for fermions are collected. In subsection 2.1 the questions of the expansion in orthogonal polynomials are discussed. The Maple procedures for the calculation of quadratically optimized polynomials and for obtaining the optimal orderings of their roots are considered in section 3 , together with a few examples. Possible generalizations of these results are discussed in section 6 . These include the polynomials needed in the second (correction-) step of the fermion algorithm and also some other cases useful for other variants of multi-bosonic algorithms. Some tests are included in section 5, together with comparisons to the minimax approximation in the special case of the function $x^{-1}$. The last section contains a summary and concluding remarks.

\section{Definitions and some basic properties}

Quadratically optimized approximations of a function $f(x)$ by polynomials $P(x)$ in an interval $x \in[\epsilon, \lambda]$ can be defined by minimizing the integral

$$
\int_{\epsilon}^{\lambda} d x[(f(x)-P(x)) w(x)]^{2} .
$$

Here $w(x)$ is an arbitrary weight function. Minimizing the relative deviation means to choose $w(x)=1 / f(x)$. Here we shall first mainly consider some negative power $f(x) \equiv$ $x^{-\alpha}$ and a positive interval $0 \leq \epsilon<\lambda$. (The required generalization to $f(x) \equiv x^{-\alpha} / \bar{P}(x)$ and more general regions will be discussed in section 6 .) In the multi-bosonic algorithm for fermions we need $\alpha=\frac{1}{2} N_{f}$, with $N_{f}$ the number of identical fermion flavours. For

numerical simulations with gluinos, which are Majorana fermions, we have $N_{f}=\frac{1}{2}$. In 
QCD $N_{f}=1,2,3$ are relevant, depending on the assumptions made on the values of quark masses.

Therefore, we shall minimize the relative deviation norm

$$
\delta \equiv\left\{(\lambda-\epsilon)^{-1} \int_{\epsilon}^{\lambda} d x\left[1-x^{\alpha} P(x)\right]^{2}\right\}^{\frac{1}{2}} .
$$

The normalization factor in front of the integral is, of course, not necessary. It is introduced for convenience. Taking the square root is also a matter of convention.

$\delta^{2}$ is a quadratic form in the coefficients of the polynomial which can be straightforwardly minimized. Let us denote the polynomial corresponding to the minimum of $\delta$ by

$$
P_{n}(\alpha ; \epsilon, \lambda ; x) \equiv \sum_{\nu=0}^{n} c_{n \nu}(\alpha ; \epsilon, \lambda) x^{n-\nu} .
$$

Performing the integral in $\delta^{2}$ term by term we obtain

$$
\delta^{2}=1-2 \sum_{\nu=0}^{n} c_{\nu} V_{\nu}^{(\alpha)}+\sum_{\nu_{1}, \nu_{2}=0}^{n} c_{\nu_{1}} M_{\nu_{1}, \nu_{2}}^{(\alpha)} c_{\nu_{2}},
$$

where

$$
\begin{gathered}
V_{\nu}^{(\alpha)}=\frac{\lambda^{1+\alpha+n-\nu}-\epsilon^{1+\alpha+n-\nu}}{(\lambda-\epsilon)(1+\alpha+n-\nu)}, \\
M_{\nu_{1}, \nu_{2}}^{(\alpha)}=\frac{\lambda^{1+2 \alpha+2 n-\nu_{1}-\nu_{2}}-\epsilon^{1+2 \alpha+2 n-\nu_{1}-\nu_{2}}}{(\lambda-\epsilon)\left(1+2 \alpha+2 n-\nu_{1}-\nu_{2}\right)} .
\end{gathered}
$$

Note that the dependence of $V$ and $M$ on $n$ comes only from the dimensions. This can be made explicit by introducing in (3) the coefficients $\tilde{c}_{n \nu} \equiv c_{n, n-\nu}$. Then everywhere in (5) we replace $(n-\nu) \rightarrow \nu$.

The coefficients of the polynomial corresponding to the minimum of $\delta^{2}$, or of $\delta$, are

$$
c_{\nu} \equiv c_{n \nu}(\alpha ; \epsilon, \lambda)=\sum_{\nu_{1}=0}^{n} M_{\nu \nu_{1}}^{(\alpha)-1} V_{\nu_{1}}^{(\alpha)} .
$$

The value at the minimum is

$$
\delta^{2} \equiv \delta_{n}^{2}(\alpha ; \epsilon, \lambda)=1-\sum_{\nu_{1}, \nu_{2}=0}^{n} V_{\nu_{1}}^{(\alpha)} M_{\nu_{1}, \nu_{2}}^{(\alpha)-1} V_{\nu_{2}}^{(\alpha)} .
$$

Scaling the integration variable $x$ by $x^{\prime}=\rho x$ one obtains the following scaling properties of the optimized polynomials:

$$
\begin{gathered}
\delta_{n}^{2}(\alpha ; \epsilon \rho, \lambda \rho)=\delta_{n}^{2}(\alpha ; \epsilon, \lambda) \\
P_{n}(\alpha ; \epsilon \rho, \lambda \rho ; x)=\rho^{-\alpha} P_{n}(\alpha ; \epsilon, \lambda ; x / \rho), \quad c_{n \nu}(\alpha ; \epsilon \rho, \lambda \rho)=\rho^{n-\nu-\alpha} c_{n \nu}(\alpha ; \epsilon, \lambda) .
\end{gathered}
$$


This allows for only considering, for instance, the standard intervals $[\epsilon / \lambda, 1]$ and obtain other cases by these scaling relations.

In applications to multi-bosonic algorithms for fermions the decomposition of the optimized polynomials as a product of root-factors is needed. This can be written as

$$
P_{n}(\alpha ; \epsilon, \lambda ; x)=c_{n 0}(\alpha ; \epsilon, \lambda) \prod_{j=1}^{n}\left[x-r_{n j}(\alpha ; \epsilon, \lambda)\right] .
$$

The scaling properties here are:

$$
c_{n 0}(\alpha ; \epsilon \rho, \lambda \rho)=\rho^{n-\alpha} c_{n 0}(\alpha ; \epsilon, \lambda), \quad r_{n j}(\alpha ; \epsilon \rho, \lambda \rho)=\rho^{j} r_{n j}(\alpha ; \epsilon, \lambda) .
$$

The numerical calculation of roots will be discussed in the next section. Since the coefficients of the polynomials are real, the roots are either real or occur in complex conjugate pairs. In the present case, for $n=$ even the roots always occur in pairs with non-zero imaginary parts. For $n=$ odd there is a single real root above the upper limit of the interval $\lambda$, and the other roots are in complex conjugate pairs.

The above formulae also apply in the limit $\epsilon \rightarrow 0$. Other generalizations will be discussed in section 国.

From the minimum property of the optimized polynomials and from the positivity of the integrand in eq. (2) one can derive interesting inequalities for the relative deviation norm $\delta$. For $0 \leq \epsilon^{\prime}<\epsilon$ we obtain

$$
(1-\epsilon) \delta_{n}^{2}(\alpha ; \epsilon, 1)<\left(1-\epsilon^{\prime}\right) \delta_{n}^{2}\left(\alpha ; \epsilon^{\prime}, 1\right) .
$$

Of course, for $m>n$ we also have $\delta_{m}(\alpha ; \epsilon, \lambda)<\delta_{n}(\alpha ; \epsilon, \lambda)$. One can also prove

$$
\delta_{n}^{2}(\alpha ; 0,1)-\epsilon<(1-\epsilon) \delta_{n}^{2}(\alpha ; \epsilon, 1),
$$

and for integer $k \geq 2$ and large enough $n$

$$
\delta_{k n}^{2}(k \alpha ; \epsilon, \lambda)<k^{2} \delta_{n}^{2}(\alpha ; \epsilon, \lambda) .
$$

In general, it does not seem to be possible to derive explicit formulae for $\delta$. In some special cases, however, explicit algebraic calculations in Maple give interesting illustrative results. For instance, in the important special case $\alpha=\frac{1}{2}$, we have

$$
\begin{gathered}
\delta_{4}^{2}\left(\frac{1}{2} ; \epsilon^{2}, 1\right)= \\
\frac{\left(\epsilon^{10}+20 \epsilon^{9}+105 \epsilon^{8}+320 \epsilon^{7}+580 \epsilon^{6}+720 \epsilon^{5}+580 \epsilon^{4}+320 \epsilon^{3}+105 \epsilon^{2}+20 \epsilon+1\right)(\epsilon-1)^{10}}{121(\epsilon+1)^{10}\left(\epsilon^{8}+24 \epsilon^{6}+76 \epsilon^{4}+24 \epsilon^{2}+1\right)\left(\epsilon^{2}+1\right)} .
\end{gathered}
$$

In the limit $\epsilon \rightarrow 0$ we have, for any $\alpha$ and at least for a wide range of orders $n$ where explicit calculation could be performed,

$$
\delta_{n}(\alpha ; 0,1)=\frac{\alpha}{n+1+\alpha} .
$$




\subsection{Expansion in orthogonal polynomials}

A useful representation of the quadratically optimized polynomials is an expansion in suitably defined orthogonal polynomials. If the relative deviation from the function $f(x)=$ $x^{-\alpha} / \bar{P}(x)$ is minimized, the appropriate integration weight in (1) is

$$
w(x)^{2}=\frac{1}{f(x)^{2}}=\left[x^{\alpha} \bar{P}(x)\right]^{2} .
$$

In most cases this is the preferred choice for the weight function. Nevertheless, also other cases are of interest, for instance $w(x)=1$ and $f(x)=x^{\alpha} \bar{P}(x)$, as in eqs. (58)-(59) of ref. [3]. Therefore, let us leave for the moment $w(x)$ and $f(x)$ general.

Let us define the orthogonal polynomials $\Phi_{\nu}(x)$ of order $\nu=0,1,2, \ldots$ such that they satisfy

$$
\int_{\epsilon}^{\lambda} d x w(x)^{2} \Phi_{\mu}(x) \Phi_{\nu}(x)=\delta_{\mu \nu} q_{\nu}
$$

The arbitrary normalization factor $q_{\nu}$ will be chosen later on by convenience. The orthogonal polynomials can be used, instead of the simple powers in (3), as a basis for the expansion of the optimized polynomials:

$$
P_{n}(\alpha ; \epsilon, \lambda ; x)=\sum_{\nu=0}^{n} d_{n \nu}(\alpha ; \epsilon, \lambda) \Phi_{\nu}(x)
$$

The advantage of this expansion is that now, as one can easily see, the matrix corresponding to $M^{(\alpha)}$ in (5) is diagonal and the coefficients of the optimized polynomial are simply given by

$$
d_{n \nu}(\alpha ; \epsilon, \lambda) \equiv d_{n}(\alpha ; \epsilon, \lambda)=\frac{b_{\nu}}{q_{\nu}}
$$

where

$$
b_{\nu} \equiv \int_{\epsilon}^{\lambda} d x w(x)^{2} f(x) \Phi_{\nu}(x) .
$$

An important property of this expansion is that, as shown by the notation, the expansion coefficients $d_{n \nu}=d_{\nu}$ do not depend on the order of the optimized polynomial $n$.

The relations in eqs. (17)-(20) show that (18) is the truncated expansion of the function $f(x)$ in terms of the basis defined by $\Phi_{\nu}(x)$ in the Hilbert space of square integrable functions in the interval $[\epsilon, \lambda]$ with integration measure $d x w(x)^{2}$. A general consequence is that for $n \rightarrow \infty$ the approximation is exponential, if the function is bounded as, for instance, $f(x)=x^{-\alpha} / \bar{P}(x)$ for $\epsilon>0$.

One can easily show that the minimum of the relative deviation norm in (7), for simplicity in the case $w(x)=1 / f(x)$, can now be expressed as

$$
\delta_{n}^{2}=(\lambda-\epsilon)^{-1} \int_{\epsilon}^{\lambda} d x\left[1-w(x) \sum_{\nu=0}^{n} d_{\nu} \Phi_{\nu}(x)\right]^{2}
$$




$$
=(\lambda-\epsilon)^{-1} \int_{\epsilon}^{\lambda} d x\left[1-w(x) \sum_{\nu=0}^{n} d_{\nu} \Phi_{\nu}(x)\right]=1-(\lambda-\epsilon)^{-1} \sum_{\nu=0}^{n} d_{\nu} b_{\nu} .
$$

For the determination of the orthogonal polynomials $\Phi_{\nu}(x)$ one can use recurrence relations [5]. Let us define the expansion of $\Phi_{\mu}(x)$ in simple powers by

$$
\Phi_{\mu}(x)=\sum_{\nu=0}^{\mu} f_{\mu \nu} x^{\mu-\nu}
$$

The up to now arbitrary normalization factor $q_{\nu}$ in (17) can be fixed by requiring

$$
f_{\mu 0}=1, \quad(\mu=0,1,2, \ldots) .
$$

The first two polynomials with $\mu=0,1$ are:

$$
\Phi_{0}(x)=1, \quad \Phi_{1}(x)=x+f_{11}=x-\frac{s_{1}}{s_{0}},
$$

with the notation

$$
s_{\mu} \equiv \int_{\epsilon}^{\lambda} d x w(x)^{2} x^{\mu}
$$

The value of the coefficient $f_{11}$ is determined by the requirement of orthogonality of $\Phi_{0}(x)$ and $\Phi_{1}(x)$. The normalization factors $q_{0}$ and $q_{1}$ are given by

$$
q_{0}=s_{0}, \quad q_{1}=s_{2}-\frac{s_{1}^{2}}{s_{0}} .
$$

The higher order polynomials $\Phi_{\mu}(x)$ for $\mu=2,3, \ldots$ can be obtained from the threeterm recurrence relation

$$
\Phi_{r+1}(x)=\left(x+\beta_{r}\right) \Phi_{r}(x)+\gamma_{r-1} \Phi_{r-1}(x) . \quad(r=1,2, \ldots) .
$$

This relation follows from the fact that $\Phi_{r+1}(x)-x \Phi_{r}(x)$ is a polynomial of order $r$, which is orthogonal to $\Phi_{r-2}(x), \Phi_{r-3}(x), \ldots$ Multiplying eq. (27) by $w(x)^{2} \Phi_{r}(x)$ and $w(x)^{2} \Phi_{r-1}(x)$ and integrating for $x \in[\epsilon, \lambda]$ we obtain for the recurrence coefficients

$$
\beta_{r}=-\frac{p_{r}}{q_{r}}, \quad \gamma_{r-1}=-\frac{q_{r}}{q_{r-1}}
$$

where

$$
p_{\nu} \equiv \int_{\epsilon}^{\lambda} d x w(x)^{2} \Phi_{\nu}(x)^{2} x
$$

Combining eqs. (17) and (29) with (22) and (25) we also obtain

$$
p_{\mu}=\sum_{\nu_{1}, \nu_{2}=0}^{\mu} f_{\mu \nu_{1}} f_{\mu \nu_{2}} s_{1+2 \mu-\nu_{1}-\nu_{2}}, \quad q_{\mu}=\sum_{\nu_{1}, \nu_{2}=0}^{\mu} f_{\mu \nu_{1}} f_{\mu \nu_{2}} s_{2 \mu-\nu_{1}-\nu_{2}} .
$$

From the recurrence relation (27), together with eqs. (28) and (30), one can recursively determine the coefficients of the orthogonal polynomials $f_{\mu \nu}$. Calculating the expansion coefficients of the quadratically optimized polynomials from eqs. (18)-(20) one obtains the desired polynomial approximation, without the knowledge of roots. Besides the representation by a product of root-factors in (9), this offers an alternative recursive way for the evaluation of optimized polynomials. 


\section{Algorithm in Maple and examples}

The calculation of the coefficients of optimized polynomials and their roots from the formulae of the previous section is, in principle, straightforward. The problem is, however, that for high orders $n=\mathcal{O}(100)$ the rounding errors in floating point calculations become dangerous. This holds for the determination of the inverse matrix $M^{(\alpha)-1}$ in eq. (6), for finding the roots of the polynomial required in (9) and for obtaining the coefficients in the recursion relation in eq. (27). Once the roots are found to sufficient precision, another problem is that applying the product representation (9), with $x$ replaced by the (squared) fermion matrix, precision losses occur again. This is because the intermediate results can be widely different in magnitude for different parts of the eigenvalue spectrum. A remedy is to find an optimized ordering of roots for the product of root-factors. All these problems can be dealt with by procedures written in an algebraic manipulation program as Maple V.

- Inversion and finding the roots: The inversion of the matrix defined in (5) can be done by the procedure linalg[inverse] included in the Maple V library. For the calculation of roots it is more convenient to write a special procedure rather than to use the Maple V library. The Laguerre iteration algorithm [7] is well suited and can be easily implemented. In the examples explicitly considered in this paper the coefficients of the polynomials are real. This could, in principle, be used to accelerate the root-finding algorithm but, for keeping the procedure general, it is better not to use the realness of the coefficients. This also allows for checking the precision by looking whether the roots are either real or occur in complex conjugate pairs, as they should. The necessary number of digits for calculating the roots is usually smaller than $2 n$. It does not depend much on the value of the power $\alpha$. (In the tests mainly $\alpha=1, \frac{1}{2}, \frac{1}{4}$ have been considered.) The precision requirements for matrix inversion and for finding the roots are roughly similar.

The calculation on typical workstations for the interesting orders up to, say, $n=100$ can be performed within several hours with storage of several 10 Mbytes. Also higher orders with $n$ equal to several hundreds are feasible with reasonable computational effort: one has to have in mind that once the optimized polynomials are found they will typically be used in fermionic Monte Carlo simulation runs lasting up to several months on the largest supercomputers.

- Optimized ordering of roots: The product representation (9), with $x$ replaced by the squared fermion matrix $X^{2}$, has to be applied to some vectors during the Monte Carlo simulations. This has to be done with care because of possible precision losses. In order to allow for a correct evaluation with 64-bit (or even 32-bit) floating point 
arithmetics, one has to choose the ordering of the factors judiciously. Since in the intermediate steps of an iterative evaluation the vector components are collected as linear combinations of several terms, precision losses can occur when different parts of the eigenvalue spectrum are multiplied by widely different values of the partial products. This can be to a large extent avoided by optimizing the ordering of roots.

It turned out that a good choice is to minimize the maximal ratio of the values $x^{\alpha} P_{p}(x)$ for $x \in[\epsilon, \lambda]$, with $P_{p}(x)$ denoting the value of the partial product under consideration. In practice this can be achieved by chosing a discrete number of points $\left\{x_{1}, x_{2}, \ldots, x_{N}\right\}$ in the interval $[\epsilon, \lambda]$ and, in a given step, comparing the values of $x^{\alpha} P_{o}(x)\left(x-r_{j}\right)$ for different choices of the next $r_{j} .\left(P_{o}(x)\right.$ is the optimized partial product obtained in the previous step.) The next root $r_{j}$ is chosen such that the maximal ratio of the values of $x^{\alpha} P_{o}(x)\left(x-r_{j}\right)$ for $x \in\left\{x_{1}, x_{2}, \ldots, x_{N}\right\}$ be minimal. The number of equidistant optimization points has been chosen typically to be $N=\mathcal{O}(n)$. For $\alpha=\frac{1}{2}, \frac{1}{4}$ and $n \simeq 100-200$ even $N \simeq n / 3-n / 5$ turned out to be sufficient.

For some purposes it is better to hold complex conjugate root pairs together in the ordering. This is the case, for instance, if the splitting up of the polynomial in two complex conjugate halves is used, as discussed in section 5. This ordering is somewhat worse for the whole polynomial, but it is necessary if the evaluation of the half-polynomials is required.

An important characteristics of this ordering principle is that the choice of the next root depends on the previously chosen ones. Generalizations are possible, for instance, to decide on the basis of the maximal ratio in the next two or three or more steps, not only in the next step alone. In this way the choice of the next root will also depend on the later choices and the optimization develops a global character.

- Recursion coefficients: The recurrence relation (27) requires to calculation of the coefficients $\beta_{r}, \gamma_{r-1}$. For this one first determines the values of the basic integrals $s_{\mu}$ in (25) and then uses eqs. (28)-(30). In this case the necessary number of digits is also high. For the simple functions $x^{-\alpha}$ it is somewhat lower than for the determination of roots, but in the more general case $x^{-\alpha} / \bar{P}(x)$ the requirements are practically the same.

An important question is the behaviour of the relative deviation norm $\delta$ as a function of the condition number $\lambda / \epsilon$ and of the order $n$. For fermionic simulations with the multi-bosonic algorithms the powers $\alpha=1, \frac{1}{2}, \frac{1}{4}$ are most interesting. I performed most of the tests with $\alpha=\frac{1}{4}$, which corresponds to the case of Majorana gluinos considered in refs. [3, 8, 9]. Several tests have also been performed with $\alpha=1$ and $\alpha=\frac{1}{2}$, which 


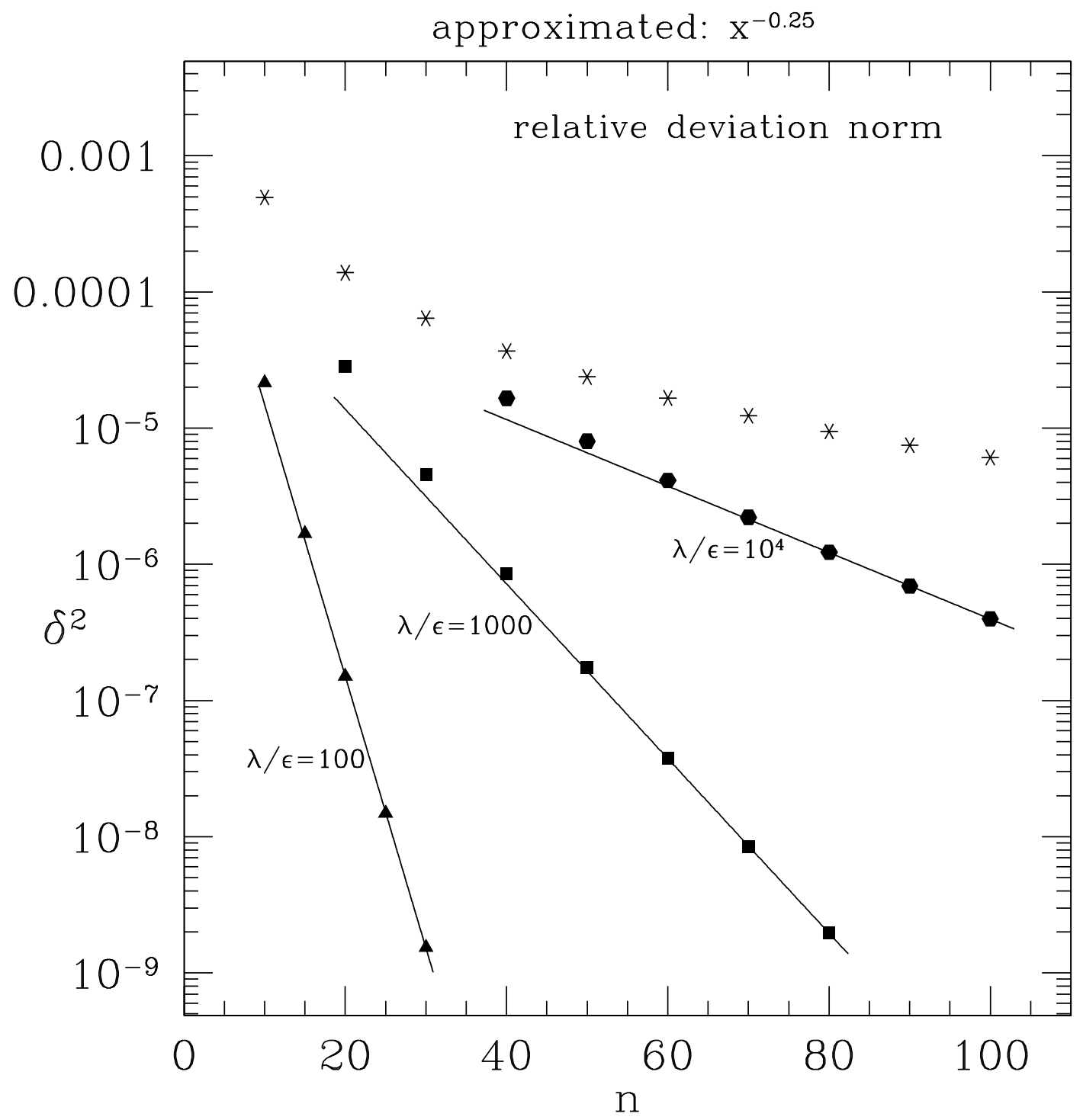

Figure 1: The (squared) deviation norm $\delta^{2}$ of the polynomial approximations of $x^{-1 / 4}$ as function of the order for different values of $\lambda / \epsilon$. The straight lines are fits to the last three points. The asterisks show the $\epsilon / \lambda \rightarrow 0$ limit given by eq. (15). 


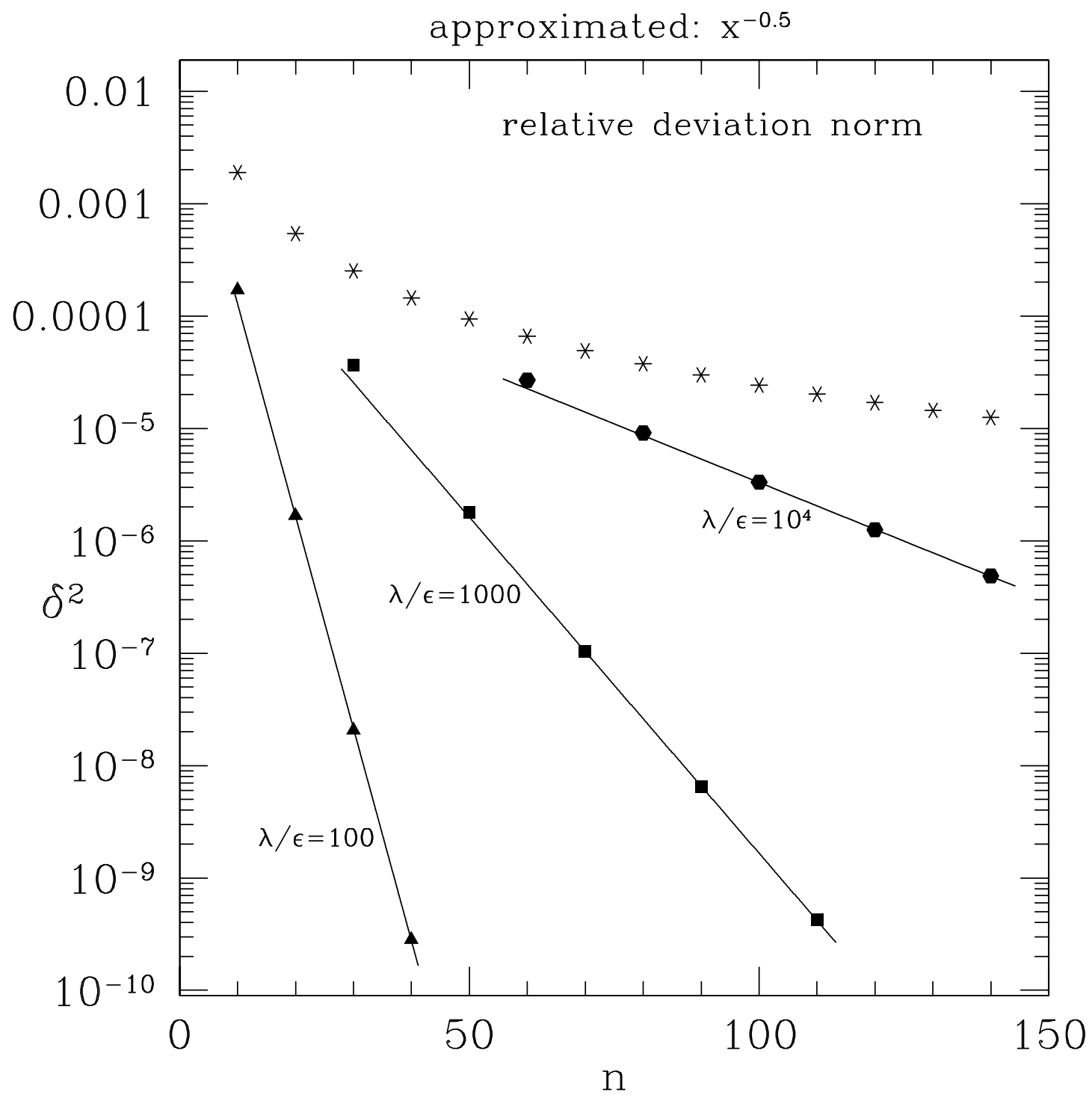

Figure 2: The (squared) deviation norm $\delta^{2}$ of the polynomial approximations of $x^{-1 / 2}$ as function of the order for different values of $\lambda / \epsilon$. The straight lines are fits to the last three points. The asterisks show the $\epsilon / \lambda \rightarrow 0$ limit given by eq. (15). 


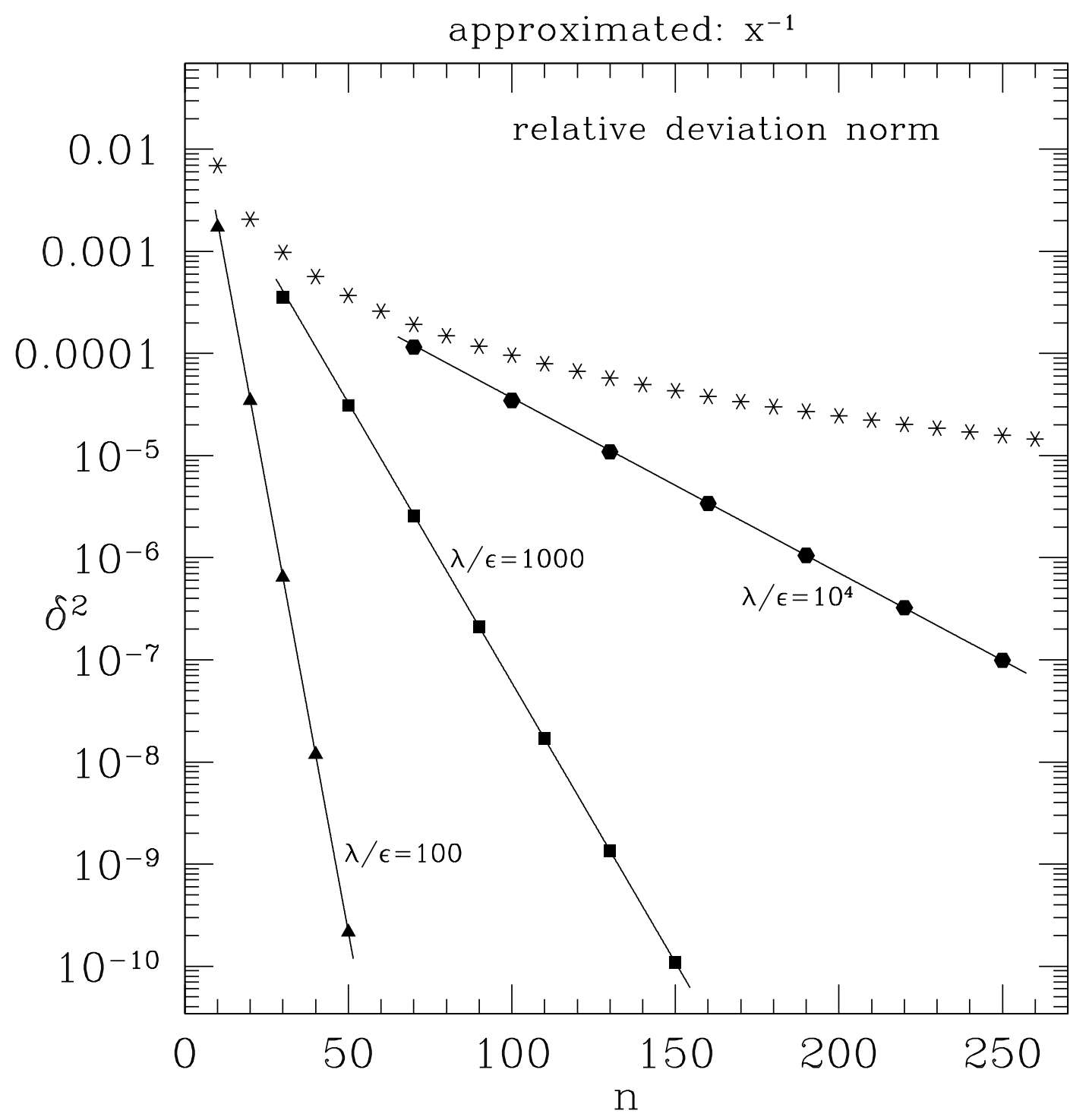

Figure 3: The (squared) deviation norm $\delta^{2}$ of the polynomial approximations of $x^{-1}$ as function of the order for different values of $\lambda / \epsilon$. The straight lines are fits to the last three points. The asterisks show the $\epsilon / \lambda \rightarrow 0$ limit given by eq. (15). 
are interesting, for instance, in numerical simulations of QCD with $2+1$ light dynamical quarks. In present day fermion simulations condition numbers up to $\lambda / \epsilon=\mathcal{O}\left(10^{4}\right)$ are in most cases sufficient.

The behaviour of $\delta^{2}$ in the interesting range of parameters is illustrated by figures 11, 2 and 3 . As is shown by the figures, the large- $n$ asymptotic behaviour of $\delta$ is well represented by the expected exponential decrease, already in the presented range or orders. In all three cases the observed behaviour is consistent with

$$
\delta_{n}(\alpha ; \epsilon / \lambda, 1) \simeq \exp \left\{-C_{\alpha} n \sqrt{\epsilon / \lambda}\right\}
$$

where the constants $C_{\alpha}$ are approximately given by $C_{1 / 4} \simeq 2.3, C_{1 / 2} \simeq 2.2$ and $C_{1} \simeq 2.0$, respectively. In fact, the fitted constants in figs. 1 and 2 are close to these values for $\lambda / \epsilon=10^{2}$ and $10^{3}$, but still about $10-20 \%$ higher for $\lambda / \epsilon=10^{4}$. In fig. 3 the asymptotic behaviour is a reasonably good representation in the whole range for all three values of $\lambda / \epsilon$.

Figures 11 to 3 also demonstrate that for relatively low orders $\delta_{n}(\alpha ; \epsilon / \lambda, 1)$ evolve close to the upper limit $\delta_{n}(\alpha ; 0,1)$ given in eq. (15). This is required by the inequality in eq. (12), as long as

$$
\frac{\alpha}{n+1+\alpha} \gg \sqrt{\epsilon / \lambda}
$$

A rough estimate for the lowest $n$ where the relatively fast exponential decrease in (31) sets in is given by the value of $n$ where the two sides of (32) become equal.

\section{Generalizations}

The great advantage of the quadratic polynomial approximation scheme defined in section 2 is its flexibility towards other functions and/or other regions of approximation. For instance, in the two-step multi-bosonic algorithm for fermions [3] three different polynomial approximations are needed: the one considered up to now with a relatively low order $\bar{n}$, denoted by $\bar{P}(x)$, and two others with higher orders, realizing a better approximation. One of them has to approximate the function $x^{-\alpha} / \bar{P}(x)$ therefore the relative deviation norm of $P(x)$ in eq. (2) is replaced by

$$
\delta \equiv\left\{(\lambda-\epsilon)^{-1} \int_{\epsilon}^{\lambda} d x\left[1-x^{\alpha} \bar{P}(x) P(x)\right]^{2}\right\}^{\frac{1}{2}}
$$

The second higher order polynomial $\tilde{P}(x)$ is similarly defined. For a possible definition see ref. [3], or alternatively, take eq. (33) with $\alpha=0$ and $\bar{P} \rightarrow P, P \rightarrow \tilde{P}$ [8].

In order to find the minimum of $\delta$ in eq. (33) one can proceed similarly to sections 2 and 3. Denoting the coefficients of the polynomial $\bar{P}(x)$ by $\bar{c}_{\bar{\nu}}$, the coefficients of the 
quadratic form in (4) are now replaced by

$$
\begin{gathered}
V_{\nu}^{(\alpha)}=(\lambda-\epsilon)^{-1} \sum_{\bar{\nu}=0}^{\bar{n}} \bar{c}_{\bar{\nu}} \frac{\lambda^{1+\alpha+\bar{n}-\bar{\nu}+n-\nu}-\epsilon^{1+\alpha+\bar{n}-\bar{\nu}+n-\nu}}{1+\alpha+\bar{n}-\bar{\nu}+n-\nu}, \\
M_{\nu_{1}, \nu_{2}}^{(\alpha)}=(\lambda-\epsilon)^{-1} \sum_{\bar{\nu}_{1}, \bar{\nu}_{2}=0}^{\bar{n}} \bar{c}_{\bar{\nu}_{1}} \bar{c}_{\bar{\nu}_{2}} \frac{\lambda^{1+2 \alpha+2 \bar{n}-\bar{\nu}_{1}-\bar{\nu}_{2}+2 n-\nu_{1}-\nu_{2}}-\epsilon^{1+2 \alpha+2 \bar{n}-\bar{\nu}_{1}-\bar{\nu}_{2}+2 n-\nu_{1}-\nu_{2}}}{1+2 \alpha+2 \bar{n}-\bar{\nu}_{1}-\bar{\nu}_{2}+2 n-\nu_{1}-\nu_{2}} .
\end{gathered}
$$

Another kind of generalization, besides (33), is to consider different integration regions. For instance, the integration region can be split in several disjoint intervals. For integer power $\alpha$ the intervals can also be extended to negative $x$. As an interesting example, let us mention the case of integer $\alpha$ with two intervals, one positive and another negative, lying symmetrically around zero. Such approximations can be useful for considering Hermitean fermion matrices with spectra symmetric around zero.

Other optimized polynomial approximations are useful in different variants of multibosonic algorithms proposed by de Forcrand et al. [10]. In particular, in the nonHermitean algorithms optimized approximations in the complex plane are required. In this case the interesting powers are twice as large as in the above Hermitean case, namely $\alpha=\frac{1}{2}$ for Majorana fermions and, in general, $\alpha=N_{f}$ for $N_{f}$ equal-mass flavours. The relative deviation norm corresponding to eq. (2) is now defined by

$$
\delta \equiv\left\{\frac{\int_{\mathcal{R}} d x d y\left|1-(x+i y)^{\alpha} P(x+i y)\right|^{2}}{\int_{\mathcal{R}} d x d y}\right\}^{\frac{1}{2}},
$$

where $\mathcal{R}$ is a region in the complex plane containing the eigenvalue spectrum of the nonHermitean fermion matrix. The coefficients of the polynomial $P(x+i y)$ are now, in general, complex. (Nevertheless, in special cases as in the explicit examples considered below, they can still be real.) The quadratic form for $\delta^{2}$ is also generally complex:

$$
\delta^{2}=1-\sum_{\nu=0}^{n}\left[c_{\nu}^{*} V_{\nu}^{(\alpha)}+V_{\nu}^{(\alpha) *} c_{\nu}\right]+\sum_{\nu_{1}, \nu_{2}=0}^{n} c_{\nu_{1}}^{*} M_{\nu_{1}, \nu_{2}}^{(\alpha)} c_{\nu_{2}},
$$

where

$$
\begin{gathered}
V_{\nu}^{(\alpha)}=\frac{\int_{\mathcal{R}} d x d y(x-i y)^{\alpha+n-\nu}}{\int_{\mathcal{R}} d x d y} \\
M_{\nu_{1}, \nu_{2}}^{(\alpha)}=\frac{\int_{\mathcal{R}} d x d y(x-i y)^{\alpha+n-\nu_{1}}(x+i y)^{\alpha+n-\nu_{2}}}{\int_{\mathcal{R}} d x d y} .
\end{gathered}
$$

For the coefficients of the optimized polynomial eq. (6) still holds.

In principle the shape of the complex region $\mathcal{R}$ can be quite arbitrary. However, for applications in multi-bosonic algorithms high polynomial orders are necessary, therefore it is advantageous to choose a region where the necessary integrals

$$
\mathcal{I}_{k_{1}, k_{2}} \equiv \int_{\mathcal{R}} d x d y(x-i y)^{k_{1}}(x+i y)^{k_{2}}
$$


with positive $k_{1}, k_{2}$, can be evaluated explicitly and the results are relatively simple. This is because the Maple procedures described in the previous section require high precision.

In case of integer power $\alpha$ rectangular or elliptical shapes are well suited. Let us first consider a rectangle $\{x \in[\epsilon, \lambda] ; y \in[-\delta, \delta]\}$ which is symmetric around the real axis. This is appropriate for fermion simulation algorithms because usually, if $\lambda$ is an eigenvalue of the fermion matrix then its complex conjugate $\lambda^{*}$ also is. The integral in (38), with positive integer $k_{1}, k_{2}$, is in this case the following:

$$
\begin{gathered}
\mathcal{I}_{k_{1}, k_{2}}=\sum_{\kappa_{1}=0}^{k_{1}} \sum_{\kappa_{2}=0}^{k_{2}}(-1)^{\frac{1}{2}\left(\kappa_{1}-\kappa_{2}\right)} \frac{\kappa_{1} !\left(k_{1}-\kappa_{1}\right) ! \kappa_{2} !\left(k_{2}-\kappa_{2}\right) !}{k_{1} ! k_{2} !} . \\
\frac{\left(\lambda^{1+k_{1}-\kappa_{1}+k_{2}-\kappa_{2}}-\epsilon^{1+k_{1}-\kappa_{1}+k_{2}-\kappa_{2}}\right)}{\left(1+k_{1}-\kappa_{1}+k_{2}-\kappa_{2}\right)} \frac{\left(\delta^{1+\kappa_{1}+\kappa_{2}}-(-\delta)^{1+\kappa_{1}+\kappa_{2}}\right)}{\left(1+\kappa_{1}+\kappa_{2}\right)} .
\end{gathered}
$$

In case of an ellipse which is symmetric around the real axis with centre at $(x=$ $\left.\frac{1}{2}(\lambda+\epsilon), y=0\right)$ and half-axes of lengths $\frac{1}{2}(\lambda-\epsilon)$ and $\delta$ in the direction of the $x$ - and $y$-axis, respectively, it is convenient to introduce the new integration variables

$$
\xi=\frac{2 x-\lambda-\epsilon}{\lambda-\epsilon}, \quad \eta=\frac{y}{\delta} .
$$

The integral is then reduced to the unit circle $\mathcal{C}$ around the origin

$\mathcal{I}_{k_{1}, k_{2}}=\frac{(\lambda-\epsilon) \delta}{2} \int_{\mathcal{C}} d \xi d \eta\left(\frac{1}{2}(\lambda+\epsilon)+\frac{1}{2}(\lambda-\epsilon) \xi-i \delta \eta\right)^{k_{1}}\left(\frac{1}{2}(\lambda+\epsilon)+\frac{1}{2}(\lambda-\epsilon) \xi+i \delta \eta\right)^{k_{2}}$

and a term-by-term evaluation gives:

$$
\begin{gathered}
\mathcal{I}_{k_{1}, k_{2}}=\frac{1}{4}(\lambda-\epsilon) \delta \sum_{i_{1}, i_{2}, i_{3}=0}^{k_{1}} \delta_{i_{1}+i_{2}+i_{3}, k_{1}} \sum_{j_{1}, j_{2}, j_{3}=0}^{k_{2}} \delta_{j_{1}+j_{2}+j_{3}, k_{2}} \\
\left(1+(-1)^{i_{2}+j_{2}}\right)\left(1+(-1)^{i_{3}+j_{3}}\right)(-1)^{\frac{1}{2}\left(i_{3}-j_{3}\right)} \frac{i_{1} ! i_{2} ! i_{3} ! j_{1} ! j_{2} ! j_{3} !}{k_{1} ! k_{2} !} . \\
\frac{\Gamma\left[\frac{1}{2}\left(1+i_{2}+j_{2}\right)\right] \Gamma\left[\frac{1}{2}\left(1+i_{3}+j_{3}\right)\right]}{\Gamma\left[1+\frac{1}{2}\left(i_{2}+j_{2}+i_{3}+j_{3}\right)\right]}\left(\frac{\lambda+\epsilon}{2}\right)^{i_{1}+j_{1}}\left(\frac{\lambda-\epsilon}{2}\right)^{i_{2}+j_{2}} \delta^{i_{3}+j_{3}} .
\end{gathered}
$$

In the complex plane, up to now, we only considered $\alpha=$ integer powers. For halfinteger

$$
\alpha \equiv A+\frac{1}{2}
$$

the evaluation of the integrals on rectangles or ellipses becomes already cumbersome. A simple possibility is to go to the square-root complex plane $(\xi, \eta)$ by the relations

$$
x+i y=(\xi+i \eta)^{2} ; \quad x=\xi^{2}-\eta^{2}, \quad y=2 \xi \eta .
$$


This transformation brings eq. (35) to the form

$$
\delta \equiv\left\{\frac{\int_{\mathcal{R}} d \xi d \eta\left(\xi^{2}+\eta^{2}\right)\left|1-(\xi+i \eta)^{1+2 A} P\left[(\xi+i \eta)^{2}\right]\right|^{2}}{\int_{\mathcal{R}} d \xi d \eta\left(\xi^{2}+\eta^{2}\right)}\right\}^{\frac{1}{2}} .
$$

Here $\mathcal{R}$ is a region in the right half of the square-root complex plane such that its image under the mapping defined by (44) covers the eigenvalue spectrum of the fermion matrix in the original $(x, y)$-plane. After this transformation one can take, for instance, rectangles or ellipses in the $(\xi, \eta)$-plane and apply formulae as (39) or (42), respectively.

Further generalizations are also possible: One can take, for instance, more complicated regions with boundaries given by polygons or ring shapes etc. Another possibility is to change the weight function $w(x)$ in the definition of the deviation norm (四)-(因). Taking e. g. $w(x)=1$ means to consider absolute deviations instead of relative ones. In multibosonic algorithms a special choice of the weight function can help to improve the quality of the approximations, for instance, by choosing $w(x)$ to be proportional to the average density of eigenvalues in the interval $[\epsilon, \lambda]$.

\section{Evaluating the polynomials: tests and comparisons}

The very high precision needed for obtaining the quadratically optimized polynomials does not mean that there is a similar difficulty in evaluating the polynomials. An extensive testing in the running project with dynamical gluinos in an $\mathrm{SU}(2)$ gauge theory showed [8, 9] that, in fact, there is no practical problem with the evaluation. Here only a small part of the performed tests are shown as represenative examples. The choice of parameters and lattice configurations is motivated by a work in progress [9]. For tests with the fermion matrix the (squared) even-odd preconditioned Hermitean fermion matrix is considered.

As a simple case, let us begin with an example for the evaluation of the polynomials on the real axis. We consider the polynomial approximation of order $n=72$ for $1 / P_{48}(x)$ in the interval $[\epsilon, \lambda]=[0.0002,3.5]$, where $P_{48}(x)$ is a 48 -th order optimized approximation of $x^{-1 / 4} / P_{16}(x)$ and $P_{16}(x)$ is a 16-th order optimized approximation of $x^{-1 / 4}$ in the same interval. The dependence of the result on the number of digits used is easily controlled by changing the Digits parameter in Maple. In figure the ratios of the results are shown for Digits $=6$ divided by Digits $=12$, which display the evaluation errors for Digits $=6$. In the left part of the figure the recursive evaluation discussed in section 2.1 is used, in the right part the root-product form in (9) with optimized ordering of roots, as described in section [3. As one can see, both evaluations give small errors of the order $\mathcal{O}\left(10^{-5}\right)-\mathcal{O}\left(10^{-4}\right)$. The errors in case of the root-product form are somewhat larger and not completely uniform in the interval. 

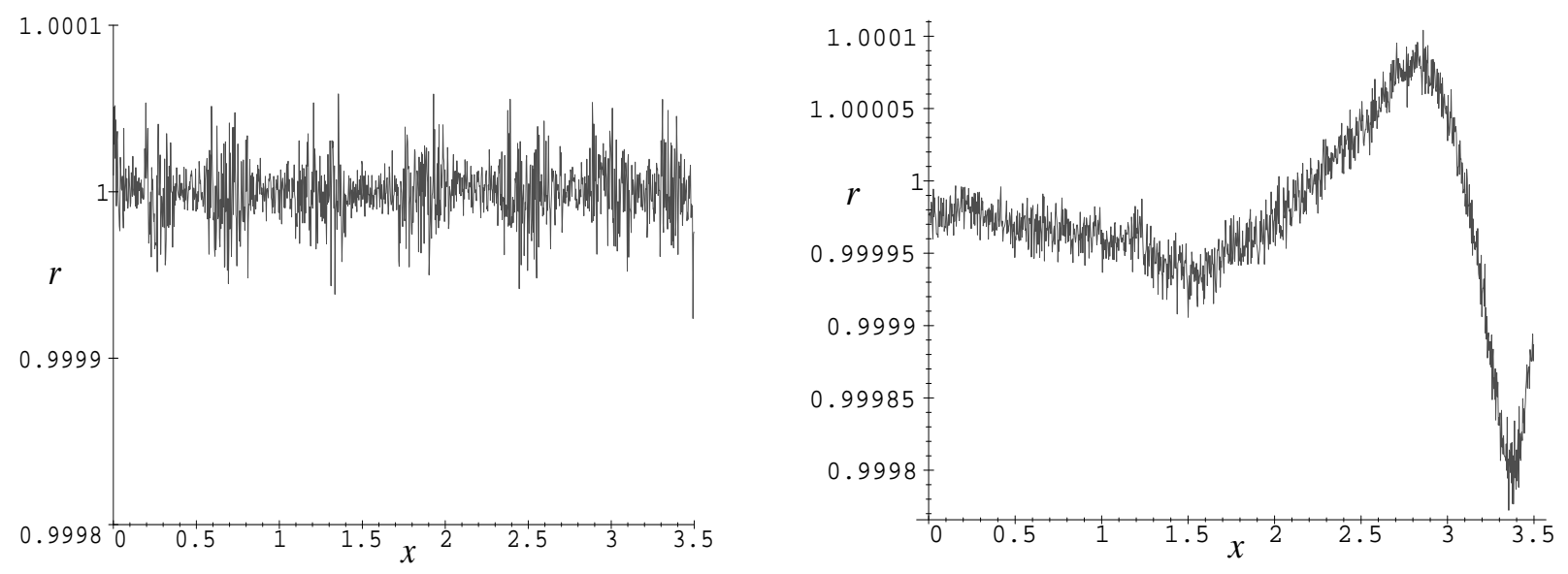

Figure 4: The rounding errors with Digits $=6$ for the evaluation of a quadratically optimized polynomial, as specified in the text, with recurrence (left) and with rootfactors in optimized order (right). (Note the small scale difference in the two halves.)

For testing the evaluation of polynomials $P\left(X^{2}\right)$ of the square of the even-odd preconditioned Hermitean fermion matrix $X$ randomly chosen configurations were taken from updating series at $\beta=2.3$ : at $K=0.185$ on $8^{3} \cdot 16$ lattice and at $K=0.190$ on $6^{3} \cdot 12$ lattice. Approximations were considered for $x^{-1 / 4}$ with order $n_{1}$, for $x^{-1 / 4} / P_{n_{1}}$ with order $n_{2}$ and for $1 / P_{n_{2}}$ with order $n_{3}$. In the two cases the orders were $\left(n_{1}=14, n_{2}=40, n_{3}=96\right)$ and $\left(n_{1}=16, n_{2}=60, n_{3}=96\right)$, respectively. The approximations were optimized in the intervals $[\epsilon, \lambda]=[0.001,3.4]$ and $[0.0002,3.5]$, respectively. The tests were carried out on the CrayT90 of HLRZ at Jülich with 64-bit arithmetics. For estimating the rounding errors the polynomials were evaluated on random Gaussian starting vectors of unit length in three different ways and the components of the differences of result vectors were considered.

The first way of evaluation was the root-product form in (9). For defining the second evaluation, the pairs of complex conjugate roots were decomposed, as usual, according to

$$
\left(X^{2}-r\right)\left(X^{2}-r^{*}\right)=\left(X-\rho_{1}\right)\left(X-\rho_{2}\right)\left(X-\rho_{1}^{*}\right)\left(X-\rho_{2}^{*}\right)
$$

and the half-polynomial

$$
P_{n}^{1 / 2}(X) \equiv \sqrt{c_{n 0}} \prod_{j=1}^{n}\left(X-\rho_{n j}\right)
$$

was taken, which gives

$$
P_{n}\left(X^{2}\right)=P_{n}^{1 / 2}(X)^{\dagger} P_{n}^{1 / 2}(X)
$$


In this second case, in the optimized ordering of roots the complex conjugate pairs were kept together, as mentioned in section 3. The third way of evaluation was the recursive one discussed in section 2.1. The performed arithmetics are in these three cases completely different, hence the rounding errors are also different. The obtained averages for the real and imaginary parts of the components of difference vectors, which would be zero for infinite precision, are shown in table 1. Since the rounding errors are independent for the pairs of evaluations considered, the individual roundind errors of an evaluation are smaller than those of the corresponding pairs. The numbers in the table show that

$$
\sigma_{1}>\sigma_{2}>\sigma_{3}
$$

In general, all the three values are comfortably small. In fact, they are of the same order as the rounding errors in global sums of order $\mathcal{O}(1)$ quantities over the lattice. The standard deviations given in the table, which were obtained by repeating the calculation 100 times with different random starting vectors, show that the rounding errors depend little on the starting vector. Similarly, the particular gauge configuration chosen from the updating did not matter either. The dependence on the polynomial order and type is illustrated on the same configurations by table 2 .

Table 1: The average value of the real and imaginary parts of components of difference vectors $\sigma_{12}, \sigma_{13}, \sigma_{23}$ for three different evaluations of the second polynomials $P_{n_{2}}$, as defined in the text. The values in the table are given in units of $10^{-12}$. The digits in parentheses give standard deviations as observed for different starting vectors.

\begin{tabular}{|c|c|c|c|}
\hline lattice & \multicolumn{1}{|c|}{$\sigma_{12}$} & $\sigma_{13}$ & $\sigma_{23}$ \\
\hline $8^{3} \cdot 16$ & $0.472(8)$ & $0.434(9)$ & $0.1569(7)$ \\
\hline $6^{3} \cdot 12$ & $0.91(7)$ & $0.88(8)$ & $0.207(1)$ \\
\hline \hline
\end{tabular}

Table 2: The same as table [ f for the three different polynomials considered. The case of root-product minus recursive evaluation is shown.

\begin{tabular}{|c|c|l|c|}
\hline lattice & $\sigma_{13}\left(P_{n_{1}}\right)$ & $\sigma_{13}\left(P_{n_{2}}\right)$ & $\sigma_{13}\left(P_{n_{3}}\right)$ \\
\hline $8^{3} \cdot 16$ & $0.0402(3)$ & $0.434(9)$ & $0.1243(4)$ \\
\hline $6^{3} \cdot 12$ & $0.0536(7)$ & $0.88(8)$ & $0.1473(9)$ \\
\hline \hline
\end{tabular}



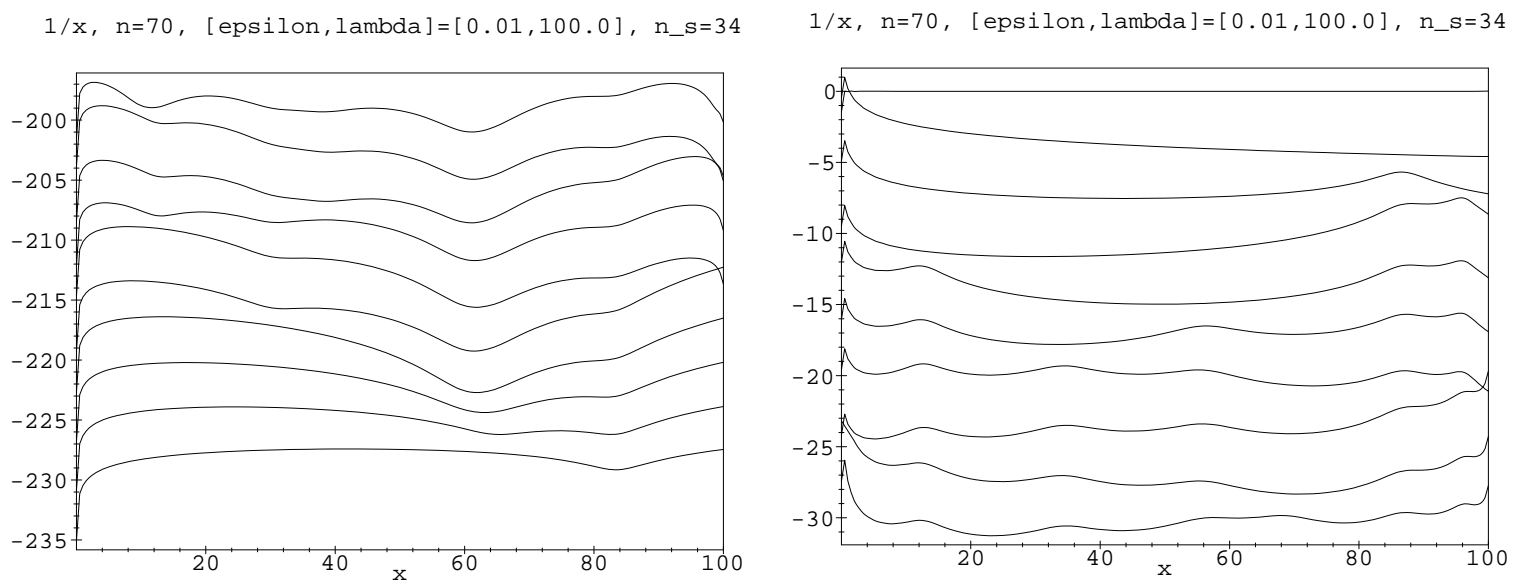

Figure 5: The evolution of the partial products towards the function $1 / x$ to be approximated in the interval $[\epsilon, \lambda]=[0.01,100.0]$. The order of the polynomial is $n=70$. The optimization of the root ordering is performed on $n_{s}=34$ points in the interval. The curves show the natural logarithms $\log \left|x P_{p}(x)\right|$ for the partial products $P_{p}(x)$ with $p=1, . ., 10$ (left) and $p=61, . ., 70$ (right).

Figure 1 and tables 11 and 2 already show that in these typical cases the achieved optimization of root orderings is completely satisfactory. In any case, the best is to use the recurrence relations and not the root-factor products, if possible. The quality of root orderings can also be demonstrated by showing the $x$-dependence of the partial products of root-factors $P_{p}(x)$ for $p=1,2, \ldots, n$. This is shown in a case by figure 5 . Remember that during the optimization the depicted curves were tried to be kept as close to horizontal lines as possible.

The third polynomial considered in the above examples (and in the two-step multibosonic algorithm) is the approximate inverse of the second one. In this case the independence of the polynomial coefficients on the final maximal order can be used to monitor the length of the residue vector of the inversion $r_{0}$ :

$$
r_{0} \equiv\left|v_{0}-P_{n_{2}}\left(X^{2}\right) P_{n_{3}^{\prime}}\left(X^{2}\right) v_{0}\right|
$$

and stop the iteration if some required precision is achieved. Here $v_{0}$ is the starting vector and $P_{n_{3}^{\prime}}$ is a polynomial with smaller order than $P_{n_{3}}$. In these tests, besides random Gaussian vectors of unit length also local starting vectors with a single non-zero component were considered from a randomly chosen site. The interval of optimization was also changed. In figure 6 , which was obtained on an $8^{3} \cdot 16$ configration at $(\beta=$ $2.3, K=0.185)$, the order of the second polynomial is $n_{2}=40$. As before, the curves 


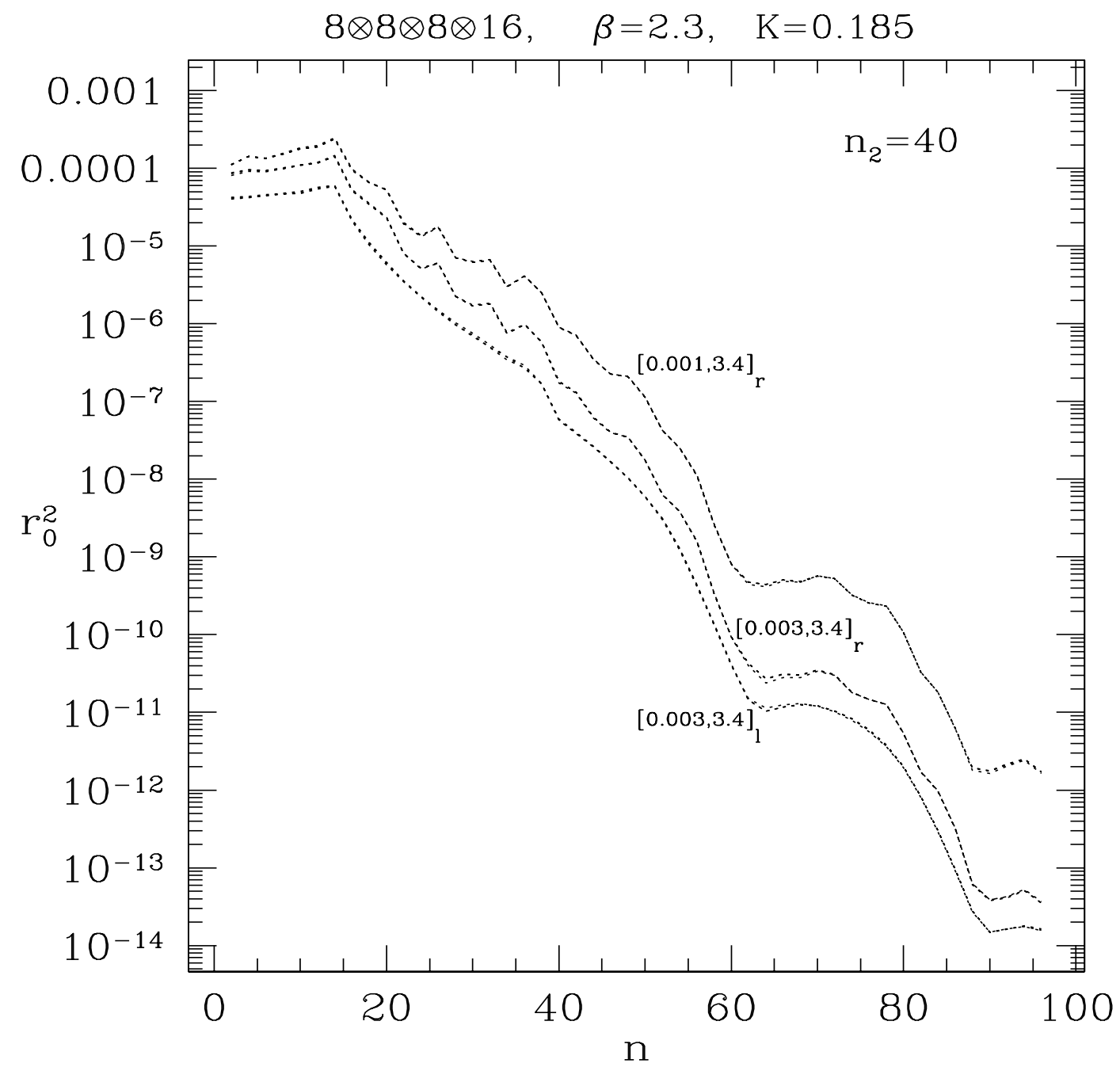

Figure 6: The length-square of the residue vector $r_{0}^{2}$ as a function of the polynomial order $n \equiv n_{3}^{\prime}$. The inverse of an optimized polynomial of order $n_{2}=40$ is calculated. The polynomials with order $n$ are optimized on the intervals $[\epsilon, \lambda]=[0.001,3.4]$ or $[0.003,3.4]$ and the starting vectors are random Gaussian $(r)$ or local from a random starting point $(l)$, as shown at the curves. 
depend only very little on the starting vector with a given type. Actually, the curves are superpositions for three different random starting vectors of the same type.

This type of inversion for polynomials with values close to one compares rather favourably with more conventional ones as, for instance, conjugate gradient iteration. (Remember that the values of $P_{n_{2}}$ are close to one because it is an approximation to $x^{-1 / 4} / P_{n_{1}}(x)$.) In fact, in order to obtain the same precision in $r_{0}$, one needs 4 CG iteration steps corresponding to $11 n_{2}=440$ matrix multiplications with $X^{2}$, as compared to $n=90$ in the figure. (Here the calculation of the residues from (50) is not counted, as it is not necessary for the inversion.)

In the special case of the function $x^{-1}$ the quadratically optimized polynomials may be compared to the Chebyshev polynomials used in [2], which minimize the maximal relative deviation. The asymptotic behaviour of $\delta$ for the Chebyshev polynomials is also given by a formula as (31) with $C_{1}=2$. However, the constants in front of the exponent are quite different. It turns out that at low orders the quadratically optimized polynomials are much better almost everywhere in the interval of approximation $[\epsilon, \lambda]$. There is only a very small piece near $\epsilon$ where the relative deviation of the Chebyshev polynomial of same order is a little bit better. This is illustrated by figure 0 . The advantage of quadratic optimization becomes larger for larger condition numbers $\lambda / \epsilon$. Concerning the roots: it turns out that in general the roots of the Chebyshev polynomials are much closer to the real axis that those of the quadratically optimized ones. For instance, in case of the polynomials in figure 7 the absolute values of the imaginary parts are an order of magnitude smaller.

Of course, if really the maximal relative deviation matters then the Chebyshev polynomials are better by definition. In the two-step multi-bosonic algorithm [3, 8, 9] there are two places where the quality of the polynomial approximations have an essential influence on the performance. First, one wants to achieve a good acceptance rate in the noisy correction step for a low order first polynomial and, second, one wants to perform the correction on random Gaussian vectors with second and third polynomials of order as low as possible. Concerning acceptance, the experience shows [9] that an acceptance about $90 \%$ can be achieved on $8^{3} \cdot 16$ lattices as a whole at large condition numbers above $10^{4}$ with a quadratically optimized first polynomial of order $n_{1} \leq 16$. In order to compare to Chebyshev polynomials, the lengths of the residue vectors

$$
r_{1} \equiv\left|v_{0}-X^{2} P_{n}\left(X^{2}\right) v_{0}\right|
$$

were considered on random Gaussian and local starting vectors from a randomly chosen site. (In both cases the starting vectors had unit length.) In order to see the dependence on the starting vector, this was repeated 100 times for different $v_{0}$. The results on $8^{3} \cdot 16$ and $6^{3} \cdot 12$ configurations are shown in table 3 and 4 , respectively. One can see that 

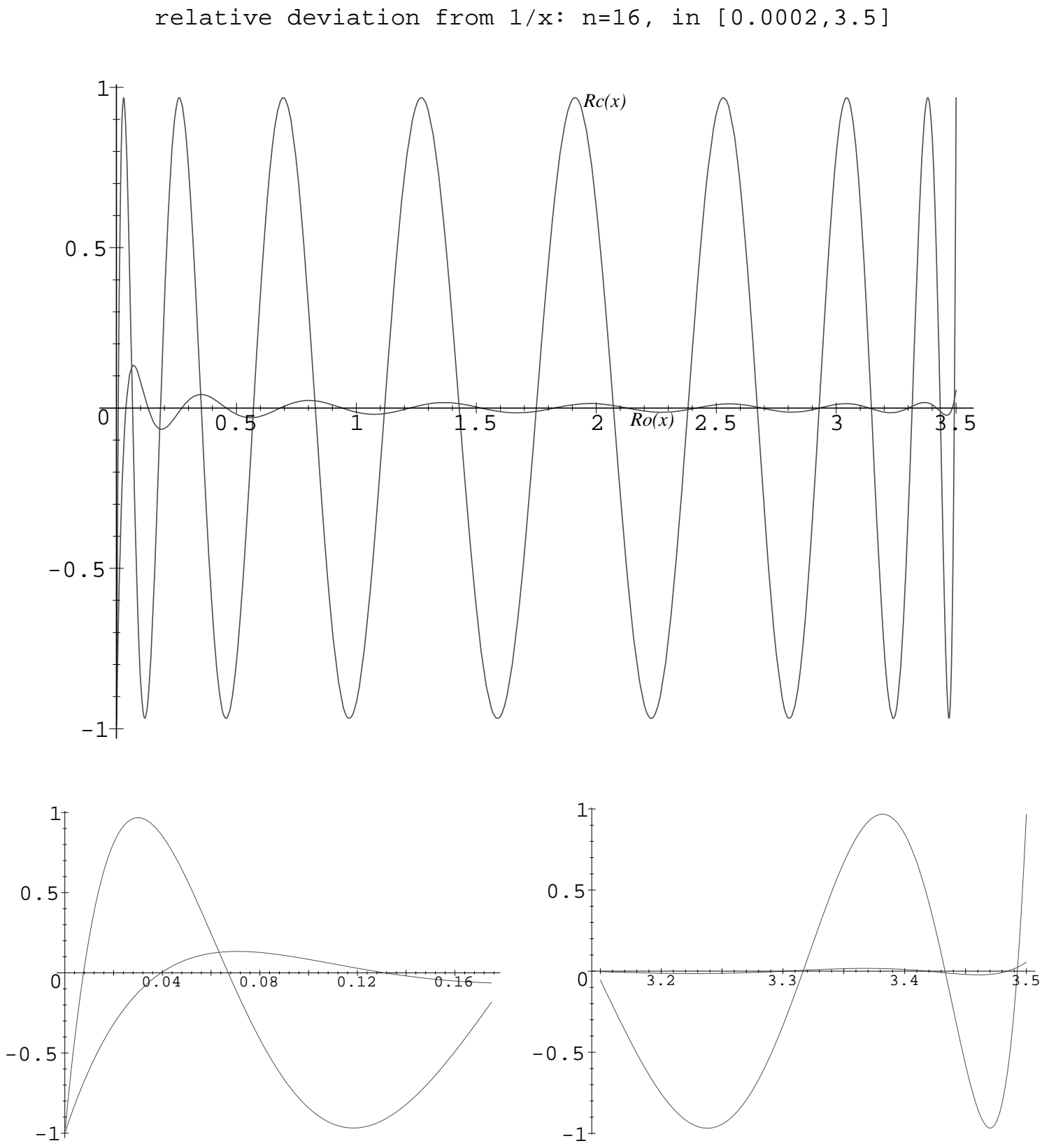

Figure 7: The comparison of the relative deviation $R(x) \equiv x P(x)-1$ for the Chebyshev polynomial $(R c(x))$ and the quadratically optimized polynomial $(R o(x))$. In the example shown the approximation interval is $[0.0002,3.5]$ and the order $n=16$ is taken in both cases. The lower part of the figure zooms on the two ends of the interval. In the left lower corner the maximum deviation of the Chebyshev polynomial is smaller: $R c(0.0002)=-0.968$ compared to $R o(0.0002)=-0.991$. 
the corresponding values for quadratic optimization are by a factor 15-200 smaller. For instance, if one would require the same residues in case of Chebyshev approximation as for the lowest order quadratic optimizations shown in the tables, one would need roughly 80 -th order instead of 14-th order in table 3 and 150-th order instead of 16-th order in table 6 . These conclusions do not depend on the particular configuration. If one picks some other gauge configurations from the updating series, the numbers in tables 3 and 4 do not change more than 20-30\% and the ratios between the left two and right two columns even less. The smaller polynomial orders result in non-trivial gains of performance in the two-step multi-bosonic algorithm, for instance, because the autocorrelations are roughly proportional to the order $n_{1}$ of the first polynomials.

Table 3: Comparison of the lengths of residue vectors $r_{1}$ defined in (51) at different orders on a $8^{3} \cdot 16$ lattice at $(\beta=2.3, K=0.185)$. The starting vectors of unit length $v_{0}$ are either random Gaussian or local with a single non-zero component from a randomly chosen site. The digits in parentheses give standard deviations as observed for different starting vectors of the same type.

\begin{tabular}{|c|l|l|l|l|}
\hline & \multicolumn{2}{|c|}{ Chebyshev } & \multicolumn{2}{c|}{ quadratic } \\
\hline order & random & local & random & local \\
\hline 14 & $0.628(1)$ & $0.441(2)$ & $0.0417(6)$ & $0.0242(12)$ \\
\hline 40 & $0.562(1)$ & $0.231(1)$ & $0.0093(2)$ & $0.00725(9)$ \\
\hline 96 & $0.0569(2)$ & $0.0359(1)$ & $0.00132(2)$ & $0.00074(3)$ \\
\hline \hline
\end{tabular}

Table 4: The same as table 3 on a $6^{3} \cdot 12$ lattice at $(\beta=2.3, K=0.190)$.

\begin{tabular}{|c|l|l|l|l|}
\hline & \multicolumn{2}{|c|}{ Chebyshev } & \multicolumn{2}{c|}{ quadratic } \\
\hline order & random & local & random & local \\
\hline 16 & $1.145(4)$ & $0.484(3)$ & $0.039(2)$ & $0.026(3)$ \\
\hline 60 & $0.813(3)$ & $0.343(2)$ & $0.0072(11)$ & $0.0051(9)$ \\
\hline 96 & $0.483(2)$ & $0.219(1)$ & $0.0025(2)$ & $0.0017(2)$ \\
\hline 192 & $0.1017(3)$ & $0.0538(4)$ & $0.00081(8)$ & $0.00050(7)$ \\
\hline \hline
\end{tabular}




\section{Summary}

In this paper the quadratically optimized polynomial approximations necessary for multibosonic algorithms of fermion simulations are considered.

In section 2 the definitions and basic properties of this scheme are introduced in a simple case, namely, optimization of the relative quadratic deviation from the function $x^{-\alpha}(\alpha>0)$ in a positive interval $x \in[\epsilon, \lambda](0 \leq \epsilon<\lambda)$. The expansion in suitably defined orthogonal polynomials is also considered. This allows for a recursive evaluation of the polynomials, without the knowledge of the roots.

An algorithm in the algebraic manipulation language Maple $\mathrm{V}$ is described in section 3 . With its help the coefficients and roots of the optimized polynomials can be determined, together with an optimal ordering of the roots for the application of the polynomial of the fermion matrix in product form with floating point arithmetics.

In section 4 generalizations are discussed which are necessary in different variants of multi-bosonic algorithms: approximations with products of polynomials and extension of the region of approximation from the real axis to the complex plane.

In general, the calculations with the given algorithms can easily be performed on modern workstations for polynomial orders as high as $n=100-300$. These are typically the maximal orders one needs in present day numerical simulations of fermionic quantum field theories. This is illustrated by figures 1, 2 2 and 3 where very good approximations with small $\delta$ are achieved already below $n=100$. Experience in SU(2) Yang-Mills theory with gluinos tells [8, 9] that for the interesting values of $\lambda / \epsilon \simeq \mathcal{O}\left(10^{3}\right)$, with rather high statistics, practically no deviation of the expectation values can be observed already for $\delta^{2} \simeq \mathcal{O}\left(10^{-6}\right)$. In these figures the simple polynomials defined in section 1 are considered, but very similar results hold also for the other two polynomials needed in the two-step multi-bosonic algorithm [3, 8], which are discussed in section 4 .

There is no principal obstacle to extend the calculations to higher orders, but then the requirements on computer power increase and further improvements of the Maple algorithms are welcome.

Detailed tests for the evaluation of the optimized polynomials are shown in section 5 in typical situations relevant in numerical simulations with dynamical fermions. These are based on experience gained in the running project with dynamical gluinos in an $\mathrm{SU}(2)$ gauge theory [8, 9]. A comparison with Chebyshev polynomials in the special case of the function $x^{-1}$ is favourable for quadratic optimization.

The main advantage of the quadratic (or least-squares) optimization is its generality and flexibility concerning the choice of functions and regions for the approximation. This is welcome in present and future large scale numerical simulations with dynamical fermions. 


\section{Acknowledgements}

It is a pleasure to thank Martin Lüscher for helpful comments. My collaborators in the dynamical gluino project, especially Klaus Spanderen and Jörg Westphalen, helped a lot with their comments. The test gauge configurations were kindly provided from running updating series by Klaus Spanderen.

\section{References}

[1] I. Montvay, G. Münster, Quantum Fields on a Lattice, Cambridge University Press, 1994.

[2] M. Lüscher, Nucl. Phys. B418 (1994) 637.

[3] I. Montvay, Nucl. Phys. B466 (1996) 259.

[4] A.D. Kennedy, J. Kuti, Phys. Rev. Lett. $\underline{54}$ (1985) 2473;

A.D. Kennedy, J. Kuti, S. Meyer, B.J. Pendleton, Phys. Rev. D38 (1988) 627.

[5] L. Fox, I.B. Parker, Chebyshev Polynomials in Numerical Analysis, Oxford University Press, 1968.

[6] B. Bunk, talk given at the conference Lattice '97 in Edinburgh.

[7] J.H. Wilkinson, Rounding Errors in Algebraic Processes, London,1963.

[8] G. Koutsoumbas, I. Montvay, A. Pap, K. Spanderen, D. Talkenberger, J. Westphalen, hep-lat/9709091.

[9] I. Montvay, K. Spanderen, J. Westphalen, in preparation.

[10] A. Borici, Ph. de Forcrand, Nucl. Phys. B454 (1995) 645; Nucl. Phys. B (Proc. Suppl.) $\underline{47}$ (1996) 800;

A. Borrelli, Ph. de Forcrand, A. Galli, Nucl. Phys. B477 (1996) 809;

A. Galli, Ph. de Forcrand, Nucl. Phys. B (Proc. Suppl.) $\underline{53}$ (1997) 956;

C. Alexandrou, A. Borici, A. Feo, Ph. de Forcrand, A. Galli, F. Jegerlehner, T. Takaishi, Nucl. Phys. B (Proc. Suppl.) $\underline{53}$ (1997) 435. 